\title{
A small dual-phase xenon TPC with APD and PMT readout for the study of liquid xenon scintillation
}

\author{
Bastian Beskers* \\ Institute of Physics \& PRISMA Cluster of Excellence, Johannes Gutenberg University, Mainz, \\ Germany \\ E-mail: beskerseuni-mainz.de
}

Pierre Sissol, Cyril Grignon, Rainer Othegraven, Melanie Scheibelhut, Uwe Oberlack

Institute of Physics \& PRISMA Cluster of Excellence, Johannes Gutenberg University, Mainz, Germany

\begin{abstract}
The MainzTPC is a small dual-phase xenon time projection chamber (TPC) with the goal to improve the understanding of response of liquid xenon to low energy elastic nuclear recoil interactions. Its design is optimized to study the charge and light yield of electronic recoils in a wide range of energies, but will also be used to measure charge and light yield of nuclear recoils. It is equipped with fast photo multiplier tubes (PMTs) and high bandwidth FADC readout to study the pulse shape of the primary scintillation light and its potential impact in Dark Matter detectors as additional background discrimination technique.

$3 \mathrm{D}$ position reconstruction is achieved by eight large area avalanche photo diodes (APDs). We report here on the design of the MainzTPC and the measurement of gain and relative quantum efficiency of the APDs.
\end{abstract}

Technology and Instrumentation in Particle Physics 2014,

2-6 June, 2014

Amsterdam, the Netherlands

\footnotetext{
* Speaker.
} 


\section{Introduction}

There is overwhelming astrophysical evidence for the existence of Dark Matter, pointing to one or more new elementary particles beyond the Standard Model. In particular, Weakly Interacting Massive Particles (WIMP) have particular theoretical appeal from a particle physics and cosmology point of view. For this reason, many experiments based on different detector materials and techniques try to detect WIMPs directly by looking at nuclear recoils produced by WIMP scattering off nucleons. In the last decade, experiments based on dual-phase xenon time-projection chambers (TPC) such as XENON10, XENON100 and most recently LUX, have set the most stringent limits in the WIMP-mass - cross-section - parameter space ([2], [4]). The principle of a dual-phase xenon TPC is schematically explained in figure 1.

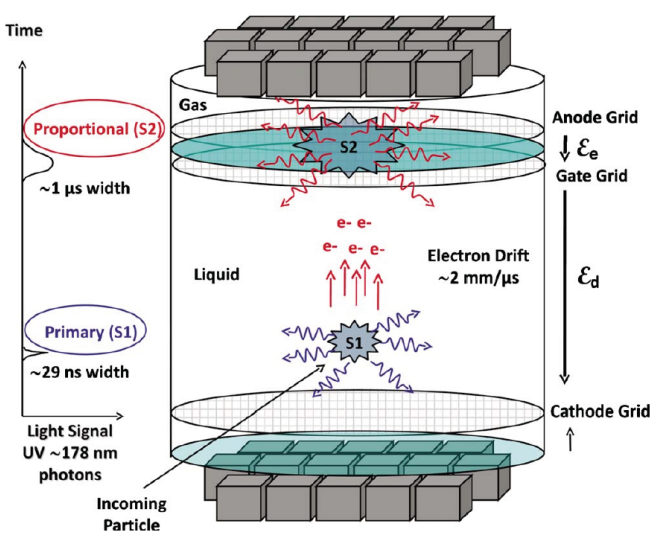

Figure 1: 1: Principle of a dual-phase LXe TPC [1].

In such dual-phase TPCs the dense liquid is used as primary detector material. A particle (e.g. WIMP) scattering off a xenon nucleus deposits a small amount of energy in the liquid leading to prompt scintillation light (S1) and ionization. The resulting free electrons are drifted though the liquid in a moderate and uniform electric field. A strong field across the liquid surface extracts the electrons into the dilute gas phase, where the field accelerates the electrons to cause a second scintillation light pulse proportional to the number of electrons, the so-called S2-signal.

Using the time difference between S1 and S2 signals and the drift velocity of electrons in liquid xenon, one can infer the depth of the interaction point (z-coordinate). The $\mathrm{x}$ - and $\mathrm{y}$-coordinates are determined from the relative intensity of the $\mathrm{S} 2$ signal on an array of light sensors on top of the TPC. The 3D-position reconstruction ability of dual-phase TPCs allows discrimination of single vs. multiple scatter interactions and definition of an optimized fiducial volume. This fiducial volume is chosen to lower the background, making use of the excellent self-shielding properties of liquid xenon, and to avoid edge effects of the detector, such as inhomogeneities of the drift field and reduced light and charge collection for interactions close to the walls of the detector.

\section{Background discrimination and scintillation pulse shape}

Despite the fiducialization and discrimination for multiple scatterings, experiments searching for WIMPs need additional background discrimination. There are two approaches to achieve background discrimination. In dual-phase TPCs the ratio S2/S1 is used. It is higher for electronic recoils from $\gamma_{\mathrm{s}}$ and electrons and lower for nuclear recoils (WIMPs, neutrons). To make use of this background discrimination technique, knowledge on the response of liquid xenon to both types of recoils and the influence of the drift field (quenching) is necessary. Other experiments based on liquid xenon chose to work with a single phase LXe and without electric field (XMASS): in this 
case there is no quenching of the scintillation light but no S2 is detected. Therefore, the S2/S1 ratio can not be used to discriminate background from signal and the pulse-shape discrimination technique is used instead. To understand the origin of the pulse shape, one has to take a closer look to the atomic processes that lead to the primary scintillation light.

After the scattering of a particle in LXe, the xenon atoms form excimers and dimers, respectively, and eventually deexcite, recombine and decay to ordinary xenon atoms. By dimer deexcitation, the primary scintillation light is produced. Different types of recoil lead to different population of singlet states (with decay time $\tau_{S}=2.2 \mathrm{~ns}$ ) and triplet states ( $\tau_{T}=27 \mathrm{~ns}$ ) of the xenon excimer, hence the shape of the primary scintillation signal S1 depends on the interaction type.

In dual-phase TPCs the use of S2/S1 is well-established and commonly used. Although the time scales are short in LXe and the electric field of the TPC reduces the number of photons generated, it could still be possible to use pulse shape discrimination as a complementary method for background discrimination. Combining the two background discrimination approaches in a liquid xenon based Dark Matter detector could significantly increase the sensitivity of the experiment by decreasing the background signal. Understanding the pulse shape is crucial to determine the requirements to a (multi-)ton scale Dark Matter detector, especially to its photo sensors and read-out electronics.

The MainzTPC has two goals: measuring charge and light yield as well as the field quenching especially in the low-energy regime (down to $\approx 2 \mathrm{keV}$ ) and improving our understanding of the primary scintillation process, i.e. the difference of the pulse shape between electronic and nuclear recoils (the population of singlet and triplet states) and the influence of the drift field.

\section{TPC design}

The design of the MainzTPC is optimized for a Compton scatter experiment. Therefore the active volume of the TPC is small ( $52 \mathrm{~mm}$ in diameter, $50 \mathrm{~mm}$ height) to avoid multiple scattering. Passive materials (especially surrounding the active volume) are kept as thin as possible, to lower the probability for $\gamma_{s}$ to scatter in non-active material. The S1 and S2 signals are detected with two cylindrical PMTs with a diameter of 2 inches, one on top and one at the bottom of the TPC. The PMTs (Hamamatsu R6041) have a high quantum efficiency at the scintillation light wavelength of $>30 \%$ and a fast response $\left(t_{\text {rise }} \approx 2 \mathrm{~ns}\right.$, TTS $\approx 0.8 \mathrm{~ns}$ ). Avoiding an array of PMTs on top has the advantage that there are no gaps, which increases the light collection efficiency of the TPC but does not allow $\mathrm{x} / \mathrm{y}$ position reconstruction of the interaction vertex. To overcome this drawback, we added an array of eight large area avalanche photodiodes around the liquid gas interface, looking inwards, to detect the S2 signal.

Geant4 simulations show, that this configuration leads to a $\mathrm{x} / \mathrm{y}$ position resolution lower than $1.3 \mathrm{~mm}$. A very uniform drift field is required not to compromise the good position resolution. For that reason the drift field is generated by fine-pitched meshes placed on top and bottom of the TPC, while the shape of the field is obtained with a flexible printed circuit board placed around the liquid xenon volume. To check the uniformity of the drift field the configuration has been simulated using COMSOL, a commercially available finite elements simulation software: the uniformity in a distance of more than $1 \mathrm{~mm}$ from the walls of the TPC is better than 1-3\%, depending on the absolute value of the field. 
The measurement of the pulse shape of small signals with short decay time constants in the nanosecond regime requires state-of-the-art electronics. We use a custom-built high bandwidth active signal splitter for the PMTs, and a high speed FADC board (Struck SIS3305, 5 GS/s, 10 bit) to digitize the S1 signals. A slower high resolution FADC board (Struck SIS3316, 16 bit, 125 MS/s) measures the S2 signal both on the PMTs and the APDs, and records the drift time. The same board also reads the charge signals of the Germanium detector, which determines the energy deposit in the liquid xenon TPC for the Compton scatter experiment.

\section{Characterization of the APDs}

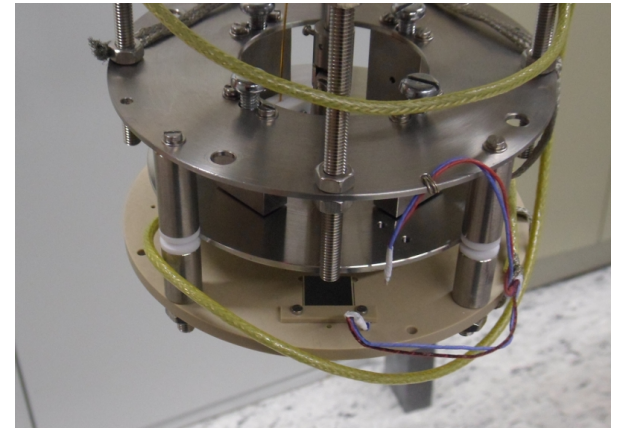

Figure 2: APD test setup

The $\mathrm{x} / \mathrm{y}$ position reconstruction of the interaction vertex requires an array of photosensors to detect the S2 signal. In the MainzTPC, this is accomplished by eight large area avalanche photo diodes (LA-APDs) surrounding the liquid-gas interface looking inwards. The choice to use APDs was driven by their compactness, a requirement to keep the TPC compact. APDs by RMD Inc. (Type S1315) were chosen, as they have been shown to be sensitive to the VUV scintillation light of liquid xenon [3], achieving a quantum efficiency of $34 \pm 5 \%$, while providing a relatively large gain of $10^{2}-10^{3}$.

The position reconstruction is based on the relative amount of light each of the APDs detects. Therefore it is necessary to know the relative quantum efficiency of the APDs and their internal gains, which are depending on temperature and bias voltage. To measure those properties a dedicated setup has been built (figure 2).

\subsection{Test Setup}

In this setup four APDs can be mounted simultaneously on a disc in a circle with the center of the APDs on a radius $r_{0}=65 \mathrm{~mm}$. Three of the APDs have been exchanged between measurement runs, and one has been kept for all runs inside the system as a reference. Above the APDs a second disc is installed, which is rotatable. On the lower side of this disc an $\alpha$-source (Am-241) is placed at a distance $r_{0}$, from the center, offering the possibility to move the source above the center of one of the APDs. The $\alpha$-particles from this source create scintillation light when the whole system is immersed in liquid xenon. This light is used to determine the relative quantum efficiency of the APDs for the xenon scintillation wavelength $(178 \mathrm{~nm})$.

Additionally, an optical fibre penetrates the rotatable disc and shines through a small piece of PTFE (thickness $1 \mathrm{~mm}$ ) which is also mounted below the rotatable disc, diffusing the light coming from the fibre. The fibre ends at $r_{\text {fibre }}=40 \mathrm{~mm}$, and therefore is not centered above the APD. The fibre is used to illuminate the APDs with pulses from a blue LED, which is placed outside and therefore kept at room temperature, even if the APDs are cooled down and immersed in liquid xenon. 
The complete system is installed in a vacuum vessel. Liquid nitrogen runs through a copper pipe surrounding the vacuum vessel, allowing to cool the system and to liquefy xenon gas. To control the temperature, three PT100 platin resistors are used. Sensor A is located outside of the vacuum vessel close to the copper pipe, sensor B is installed in the vacuum vessel close to the reference APD and sensor $\mathrm{C}$ is installed $5 \mathrm{~mm}$ above the Am-241 source. The latter being used only to check if the $\alpha$-source is immersed in liquid or not, whereas sensor B's reading is the temperature of the APD used in data analysis. Sensor A is used as input temperature for the PID control loop, regulating the LN2-flow to stabilize the temperature. This vacuum vessel (containing APDs and liquid xenon) is installed in a vacuum cryostat.

\subsection{Electronics and Readout}

For the characterization measurements, the APD signals were fed into a charge sensitive preamplifier (CAEN, A1422, 400mV/MeV). Additionally, a shaping amplifier (Ortec, Model 671) was used to shape the signal and to match the input range of the multi-channel analyzer (FastComtek, MCA-3), which we used to record the data. For the bias voltage of the APDs we used a NIM HV power supply (ISEG 234M or ISEG 206L). To monitor and control the temperature inside the chamber and do the PID control of the LN2 cooling, we used a cryo-controller (SRS CTC100) in combination with a nitrogen flow controller (MKS, M100B). To calibrate the MCA spectra and to scale spectra recorded at different gains of the shaping amplifier, a test pulse from a precision pulser (BNC, PG-5) was always recorded in parallel to the APD signals. The blue LED (370 nm) was driven by a Philips pulser (PM5786B).

\subsection{Measurement Procedure}

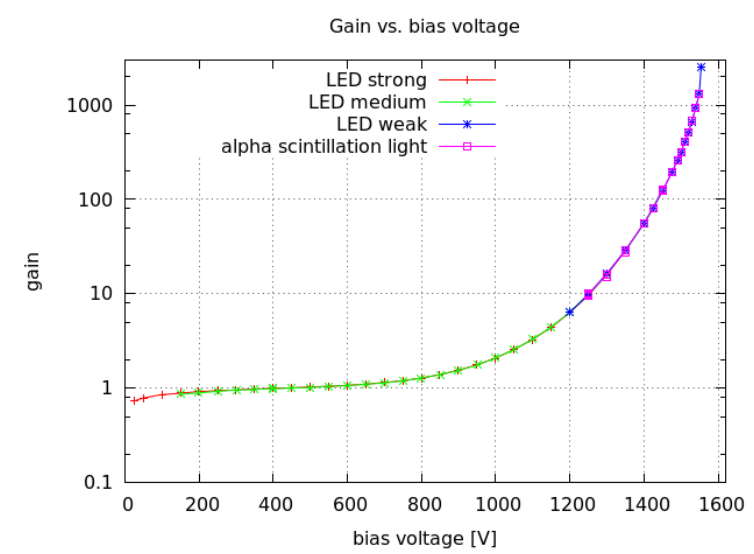

Figure 3: Gain measurement with different LED pulses and $\alpha$-scintillation light
As the multi-channel analyzer used to record the signal spectra only has one input channel, we measured one APD after the other. During each APD measurement the temperature was stable with deviations of less than $0.1 \mathrm{~K}$. In addition to the $\alpha$ scintillation light pulses, a test pulse and a LED light pulse were always recorded. To scan the whole gain range of the APDs it was necessary to change the strength of the LED light pulse, using a strong pulse for the low gain regime ( $g \approx 0.25-10$ ), a medium strong light pulse for the gain range of 1 to several 100 's and a weak LED light pulse for the highest gains. As the different light pulses need to be scaled according to their relative strength, there was an overlap between at least the highest five settings of bias voltage of the higher light pulse and the lowest five settings of the lower light pulse. Signal spectra were recorded with different bias voltages $\left(25 \mathrm{~V}<U_{b}<U_{b}^{\max }\right)$, changing the bias voltage in steps of $50 \mathrm{~V}$ in the flat regime (compare figure 3 ) and smaller steps in regions where the gain changed stronger with changing bias voltage. The smallest step size $(10 \mathrm{~V}$ 
down to $5 \mathrm{~V}$ ) was used for the highest gains or bias voltages above $1500 \mathrm{~V}$ accordingly. The maximum bias voltage used was different for different APDs and temperatures. In most cases we used $U_{b}^{\max }=1550 \mathrm{~V}$.

As the gain of the APD covers three orders of magnitude, it was not only necessary to change the strength of the LED pulses with increased bias voltage, but it was also necessary to change the gain of the spectroscopy amplifier to keep the scintillation light signals in the input range of the MCA. Each spectrum recorded this way contains therefore two peaks (test pulse and LED) for the low gain regime, where the $\alpha$-scintillation light is not visible or three peaks, if $\alpha$-light was visible. This process was repeated at least at three different temperatures between $169 \mathrm{~K}$ and $180 \mathrm{~K}$, to determine the temperature dependency of the gain in the temperature range where xenon is in liquid state.

\subsection{Data Analysis and Results}

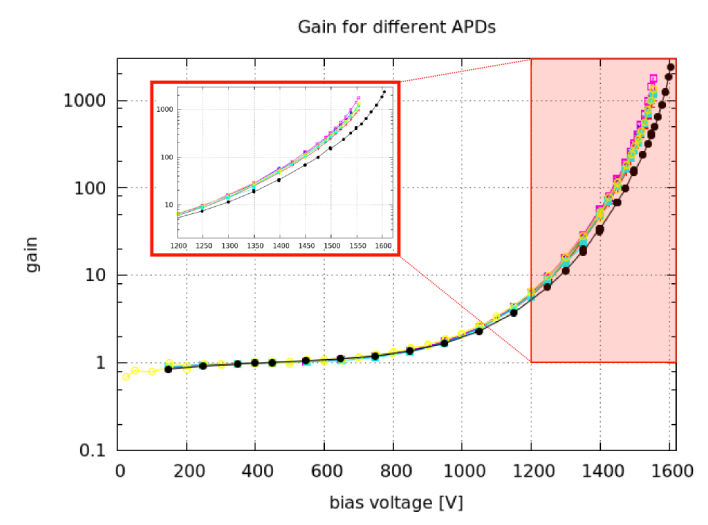

Figure 4: Gain measurement for different APDs

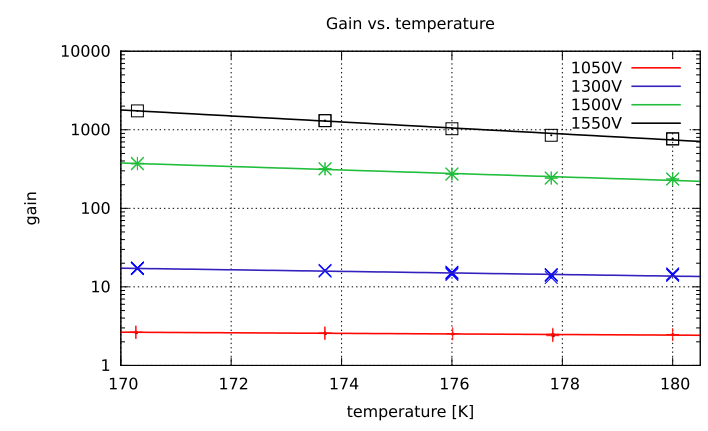

Figure 5: Temperature dependency of APD gain
For data analysis, all recorded spectra for one APD measurement run (constant temperature, scanning the whole gain range with different LED pulse strength as described above) were saved into one binary file together with supplementary information like temperature, bias voltage, spectroscopy amplifier gain, test pulse amplitude, etc. Using the peak position and the amplitude of the test pulse and the capacitance of the charge-sensitive preamplifier (CSP) test pulse input $\left(C_{T P}=1 \mathrm{pF}\right)$, the spectra could be calibrated and converted from MCA channels to charge. The mean of each peak, fitted with a Gaussian, were converted to charge too.

The amount of light produced by the different light sources (different LED pulses and scintillation light) is different. The difference between two light sources $i$ and $j$ can be described with one single factor $f_{i j}$, which was calculated as follows:

$$
f_{i j}=\frac{\sum_{n=0}^{N} \frac{Q_{i}\left(U_{b, n}\right)}{Q_{j}\left(U_{b, n}\right)}}{N}
$$

where $Q_{i}$ is the peak position of the light source $i$ and $Q_{j}$ is the peak position of light source $j$ at bias voltage $U_{b, n} . \mathrm{N}$ is the number same bias voltage.

of spectra taken with the two light sources with the same bias voltage.

Using these factors, the spectra (and fit parameters of each peak respectively) could be normalized to the strongest light source, i.e. the strongest LED pulse. To determine the gain of the 
APD, one has to specify equal gain, which was chosen arbitrarily to be at a bias voltage of $400 \mathrm{~V}$, driven by the fact, that the peak position of the LED pulse did not change much with changing bias voltage between $\approx 200 \mathrm{~V}$ and $\approx 600 \mathrm{~V}$. At bias voltages below $200 \mathrm{~V}$ the peak position of the LED pulses increases significantly, due to the incomplete charge collection in the avalanche region at too low internal fields. At voltages significantly larger than $400 \mathrm{~V}$ (i.e. around $600 \mathrm{~V}$ ) the peak position started to increase again with increasing bias voltage, showing that the avalanche process starts.

As the gain at $400 \mathrm{~V}$ was defined to be 1 , we divided the measured charge of each of the peaks by the measured charge of the same peak at $U_{b}=400 \mathrm{~V}$, using the charge scaled to the strongest LED pulse. As a result, we get the gain dependency on bias voltage for one stable temperature, as shown for the reference APD in figure 3.

In figure 4 one can see the gain of all measured APDs. The temperature was constant while measuring one APD $(\Delta T<0.1 \mathrm{~K})$, but the absolute temperature was not the same for all APDs. Temperature deviations between different APDs was $\Delta T_{\text {dif }} \pm 0.3 \mathrm{~K}$. One can see, that most APDs (colored lines) show a quite similar behaviour, but one APD (black line) clearly deviates from the others. This APD is from a different silicon wafer and might therefore have tiny differences in the internal structure.

The avalanche effect is very strongly depending on the temperature, therefore we measured gain-voltage dependency of each APD at different temperatures between $169 \mathrm{~K}$ and $180 \mathrm{~K}$. Using this data allows to plot gain vs. temperature for different bias voltages. These curves were fit with an exponential

$$
g(T)=g_{0} \cdot \exp \left(-k_{0}\left(T-T_{0}\right)\right)
$$

where $k_{0}$ is the relative change of the gain per degree Celsius. The result for the reference APD is shown for four different bias voltages, covering small gains up to the highest gain, in figure 5. The high values of $k_{0}$ clearly show, that it is inevitable to measure and control temperature in the MainzTPC accurately and include the measured temperature into the position reconstruction algorithm.

For the position reconstruction, the knowledge of relative quantum efficiency of each APD for the xenon scintillation light $(178 \mathrm{~nm})$ is crucial. The relative quantum efficiency can be calculated from the peak position of the $\alpha$-scintillation light peak. Dividing the measured charge of the scintillation light peak by the internal gain of the APD for a given bias voltage and the electron charge $e$ gives the number of detected scintillation photons $N_{p h}^{d e t}$.

$$
N_{p h}^{\text {det }}=\frac{Q_{\text {meas }}\left(U_{b}\right)}{g\left(U_{b}\right) \cdot e}
$$

This number is independent of the gain of the APD. For each APD, this number is compared to the number of photons detected by the reference APD $N_{p h}^{d e t, r e f}$, allowing to state the quantum efficiency $Q E_{r e l}$ of each APD $i$ relative to that of the reference APD.

Determination of the absolute quantum efficiency of the APDs is more difficult, as one has to know the absolute number of photons $N_{p h}^{h i t}$ that hit the APD. This number depends on the $\mathrm{W}$-value, i.e. the amount of energy from $\alpha$-particles needed to produce one scintillation photon $\left(W_{p h}=19.6 \mathrm{eV}\right.$ [1] $)$ and the fraction of produced photons that hit the APD. This fraction can be calculated by the solid angle of the APD seen from the Americium source, but additionally some of the photons that would not hit the APD directly, could be reflected on the walls and anyway hit 
the APDs and therefore increase the effective solid angle of the APDs. As we are only interested in the relative quantum efficiency of the APDs, we did not carry out the Geant 4 simualtions that would be necessary to get a handle on the reflections, but used just the geometrical solid angle of the APDs seen from the $\alpha$-source.

In addition to the geometry and the $\mathrm{W}$-value, the purity of the xenon plays an important role, when trying to infer the number of photons hitting the APD, as impurities change the number of photons created per unit of $\alpha$-particle energy as well as they might lead to absorption of VUV photons. The latter two effects are the reason why one should not take the values of absolute quantum efficiency stated in figure 6 too serious, especially as for the second and third batch of tested APDs the xenon system had a leakage and the absolute quantum efficiencies were obtained using the relative quantum efficiency of the APDs and the absolute value of quantum efficiency calculated for the reference APD for the first measurement run. Still the absolute values of QE we infered are similar to the one measured before in ([3]).

Looking at the measured relative quantum efficiencies, one can see that two APDs are significantly worse than the others. The reason is, that one of those (APD 8) was from a different silicon wafer, it is the same APD that deviates in the $U_{b}$-g-plot from the other APDs. The second "bad" APD (APD 7), was used before and might not have been treated carefully enough, so the reason for the worse QE of this APD might be some surface contamination that absorbs the VUV scintillation photons before they reach the sensitive region of the APD.

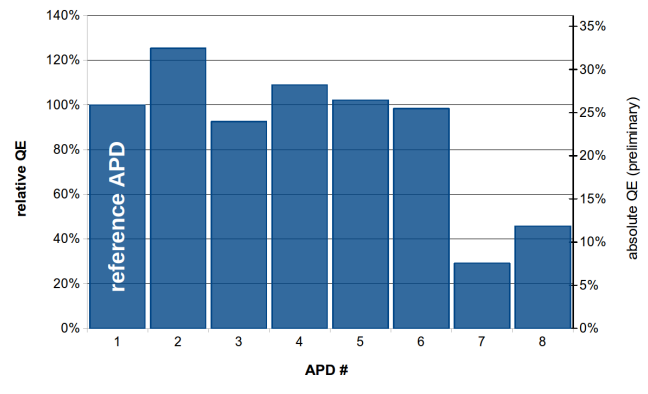

Figure 6: Quantum efficiency of the APDs relative to the reference APD

\section{Summary and Outlook}

We have designed and built a small xenon dual-phase TPC and a setup to carry out a Compton scatter experiment to study low-energy response of liquid xenon to electronic recoils with unprecedented accuracy, using a germanium detector to determine recoil energy and position resolution to fiducialize the active volume and discriminate multiple scatter events. Additionally our TPC is equipped with fast PMTs and high speed Flash-ADCs to study the scintillation light pulse shape of liquid xenon. The APDs that provide $\mathrm{x} / \mathrm{y}$-position resolution have been characterized using LED pulses and the VUV scintillation light produced by $\alpha$-particles in liquid xenon. Measurements of the gain of each of the APDs at different temperatures and bias voltages as well as the measurement of the relative quantum efficiency of the APDs enable us to do 3D-position reconstruction in the TPC as planned. We started with the assembly of the TPC and the cryo-system and look forward to see first signals this summer and to measure pulse shape as well as charge and light yield later this year. For the future, it is planned to move the TPC from Mainz to a suitable neutron source (e.g. ELBE) to study xenon response to nuclear recoils.

Applying pulse shape discrimination in a multi-ton scale detector will set strong requirements to the detector design and its photo-sensors, as photon travel times, photon reflections and response 
of the photo sensors will alter the measured pulse shape. Using the acquired knowledge on the scintillation pulse shape will enable us to study the requirements for a multi-ton detector and additional background reduction that could be achieved.

Acknowledgements This project is supported by the Helmholtz Alliance of Astroparticle Physics (HAP), the PRISMA Cluster of Excellence, and the state of Rhineland Palatinate through the EMG research center.

\section{References}

[1] E. Aprile and T. Doke, "Liquid Xenon Detectors for Particle Physics and Astrophysics", Rev. Mod. Phys. 82, 2053-2097 (2010), [arXiv: 0910.4956 [physics.ins-det]].

[2] The XENON100 Collaboration (E. Aprile et al.), "Dark Matter Results from 225 Live Days of XENON100 Data", Phys. Rev. Lett. 109, 181301 (2012), [arXiv:1207.5988v2 [astro-ph.CO]].

[3] P. Shagin et al., "Avalanche Photodiode for liquid xenon scintillation: quantum efficiency and gain", JINST 4 P01005 (2009), [doi:10.1088/1748-0221/4/01/P01005].

[4] The LUX collaboration (D.S. Akerib et al.), "First Results from the LUX Dark Matter Experiment at the Sanford Underground Research Facility ", Phys. Rev. Lett. 112, 091303 (2014),

[doi:10.1103/PhysRevLett.112.091303]. 